\title{
Topological fate of edge states of single Bi bilayer on $\mathrm{Bi}(111)$
}

\author{
Han Woong Yeom, ${ }^{1,2, *}$ Kyung-Hwan Jin, ${ }^{2}$ and Seung-Hoon Jhi ${ }^{2}$ \\ ${ }^{1}$ Center for Artificial Low Dimensional Electronic Systems, Institute for Basic Science (IBS), 77 Cheongam-Ro, \\ Pohang 790-784, Republic of Korea \\ ${ }^{2}$ Department of Physics, Pohang University of Science and Technology (POSTECH), Pohang 790-784, Republic of Korea
}

(Received 7 November 2015; revised manuscript received 16 January 2016; published 26 February 2016)

\begin{abstract}
We address the topological nature of electronic states of step edges of $\mathrm{Bi}(111)$ films by first-principles band structure calculations. We confirm that the dispersion of step-edge states reflects the topological nature of underlying films, which become topologically trivial at a thickness larger than eight bilayers. This result clearly conflicts with recent claims that the step-edge state at the surface of a bulk Bi(111) crystal or a sufficiently thick $\mathrm{Bi}(111)$ film represents nontrivial edge states of the two-dimensional topological insulator phase expected for a very thin $\mathrm{Bi}(111)$ film. The trivial step-edge states have a gigantic spin splitting of one-dimensional Rashba bands and substantial intermixing with electronic states of the bulk, which might be exploited further.
\end{abstract}

DOI: 10.1103/PhysRevB.93.075435

\section{INTRODUCTION}

The hallmark of a topological insulator (TI) is the existence of chiral edge mode(s) along edges of the material. In its two-dimensional (2D) version, the chiral edge mode is highly interesting, representing a quantum spin Hall (QSH) channel [1-4]. Very recently, such QSH edge states were reported to be directly probed by scanning tunneling microscopy and spectroscopy (STM/STS) for single Bi bilayers (BLs) grown on $\mathrm{Bi}_{2} \mathrm{Te}_{2} \mathrm{Se}[5,6]$ and graphene [7], following an earlier theoretical prediction [8,9]. The QSH phase of Bi ultrathin films was predicted to be stable for films of a few bilayers [10] and a thicker film would become topologically trivial as expected for bulk Bi crystals [11]. On the other hand, a recent STS study found edge-localized states, possibly spin polarized, along step edges at the surface of a Bi(111) crystal and related them to the topological edge state of the 2D topologically insulating (or semimetal) single Bi BL [12]. This claim was followed by a more recent work on thick $\mathrm{Bi}(111)$ films grown on a $\mathrm{Si}$ substrate [13]. That is, these works interpret the surface layer of a $\mathrm{Bi}(111)$ crystal as a $2 \mathrm{D}$ topological material in apparent contradiction to the current knowledge of a $\mathrm{Bi}$ crystal as a topologically trivial metal. This leads to important questions of whether the topologically trivial $\mathrm{Bi}(111)$ crystal is covered with a nontrivial 2D layer and how the interaction of the surface Bi layer with its bulk preserves (or affects) its topological properties.

In this work, we explicitly check the $1 \mathrm{D}$ band structure of step edges of $\mathrm{Bi}(111)$ films as a function of the film thickness. We unambiguously clarify that the $\mathrm{Bi}(111)$ surface layer on top of a sufficiently thick film becomes a trivial 2D metal, reflecting the topological phase transition of the $\mathrm{Bi}(111)$ film, and its step edge has trivial spin-split bands of the 1D Rashba type.

\section{Calculations}

$A b$ initio calculations were carried out in the planewave basis within the generalized gradient approximation

\footnotetext{
*yeom@postech.ac.kr
}

for the exchange-correlation functional using the VASP package [14,15]. A cutoff energy of $400 \mathrm{eV}$ was used for the plane-wave expansion and a $k$-point mesh of $15 \times 1 \times 1$ for the Brillouin zone sampling. The spin-orbit coupling was considered as implemented within VASP at the second variational step using the scalar-relativistic eigenfunctions as a basis [16]. In order to investigate edge states, we carried out calculations for single BL Bi nanoribbons (BNRs) of 9 or 15 Bi zigzag chains on top of $1-6$ or 7-9 BL slabs, respectively, as illustrated in Fig. 1. This mimics step edges with the zigzag structure of 2-10 BL films. The atoms of the Bi slabs and the nanoribbons on top were fully relaxed until the HellmannFeynman force was less than $0.01 \mathrm{eV} / \AA$. The topological invariants were calculated using the well-established method of Soluyanov and Vanderbilt, which can handle a system without inversion symmetry [17].

\section{RESULTS AND DISCUSSION}

For ultrathin floating $\mathrm{Bi}$ films, detailed band structure calculations are already available, which show that the 2D TI nature of Bi bilayers is maintained up to $8 \mathrm{BLs}$ [10]. Thicker films are expected to become metallic with the band gap closed, obtaining the bulk properties of a topologically trivial metal. However, this topological phase transition was apparently not clarified in previous work. Since this point is critical in the following discussion, we first check the thickness needed for the phase transition into a trivial phase. The fingerprint of a topologically nontrivial QSH phase is band inversion, which always involves the closing and reopening of the band gap as the strength of spin-orbit coupling is increased. This band-gap reopening can be seen clearly for floating $\mathrm{Bi}(111)$ films up to the thickness of 8 BLs [Figs. 2(a)-2(d)], which is consistent with the previous calculation [10]. However, from $9 \mathrm{BL}$, there exist two successive band-gap closings [Figs. 2(e) and 2(f)], which indicate a phase transition into a topological phase and a reentrant transition into a trivial phase. The trivial phase at the full strength of SOC for 9 and $10 \mathrm{BL}$ films is confirmed unambiguously by the band character with no inversion and further by the null topological invariant calculated (Table I). That is, the trivial bulk metallic behavior is reached at the thickness of $9 \mathrm{BL}$. 
(a)

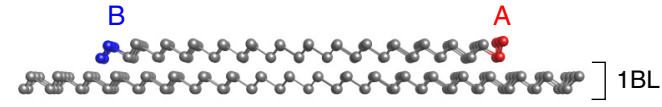

(b)

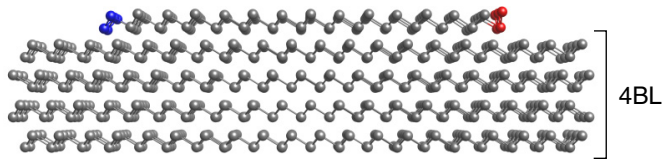

(c)

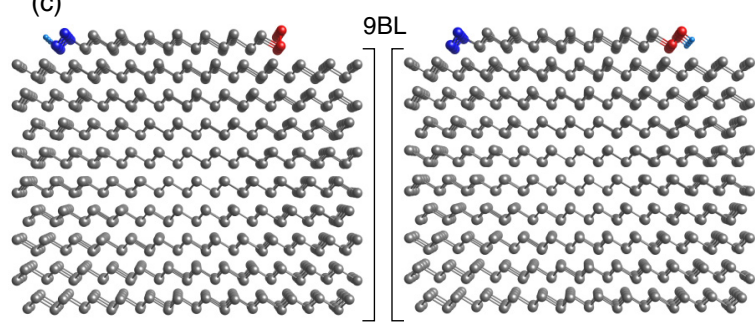

FIG. 1. Structural models of zigzag Bi nanoribbons on (a) 1, (b) 4, and (c) 9 BL Bi(111) films. Type A and type B edges are marked by red and blue atoms, respectively, which undertake different types of reconstruction. In order to reduce the computational load, we used narrower nanoribbons on $9 \mathrm{BL}$ films. In this case, one edge is passivated by $\mathrm{H}$ atoms to ensure the decoupling between the edges.

We also examined the band structure of surface layers of these films. Surface layers of a thick Bi(111) film or the bulk $\mathrm{Bi}(111)$ have topologically trivial 2D spin helical bands due to the Rashba-type spin-orbit interaction $[18,19]$. In contrast to
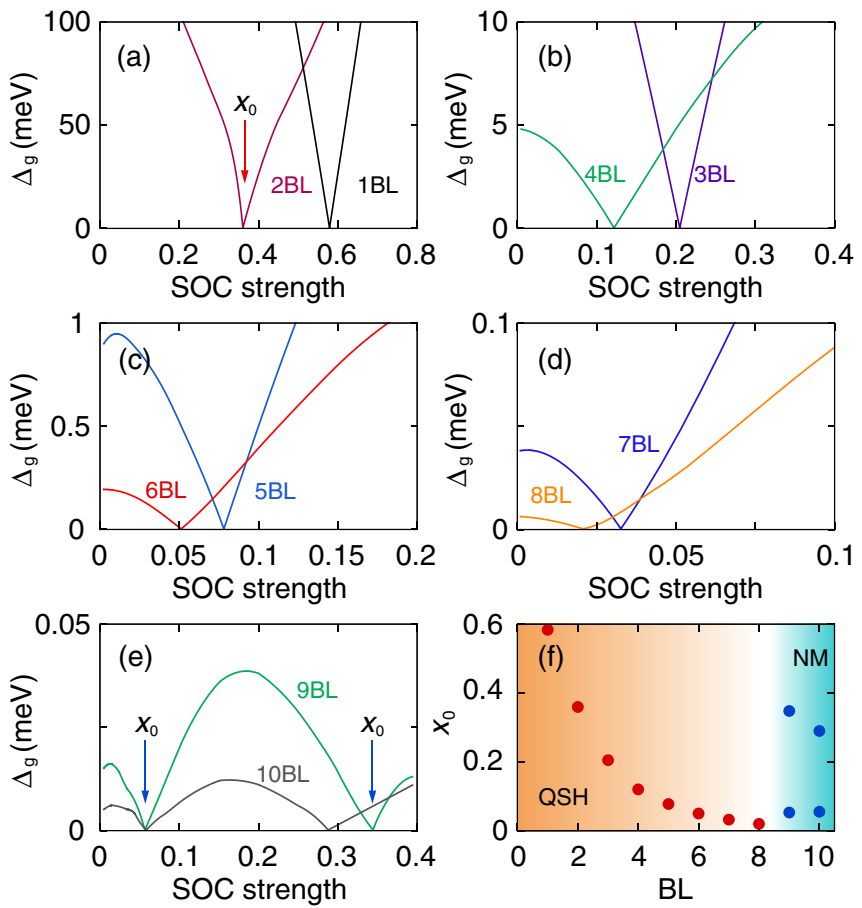

FIG. 2. (a)-(e) Calculated energy gaps $\left(\Delta_{g}\right)$ of $1-10 \mathrm{BL} \mathrm{Bi(111)}$ films at the $\Gamma$ point as the strength of spin-orbit coupling (SOC) is varied. The SOC strength 1.0 corresponds to that of the bulk $\mathrm{Bi}(111)$. The gap-closing point $\left(X_{0}\right)$ is exponentially reduced as the film thickness is increased. There are two gap-closing points above $8 \mathrm{BL}$ and the QSH phase changes into the normal metallic (NM) phase.
TABLE I. Calculated $Z_{2}$ number of $\mathrm{Bi}$ films with different thicknesses.

\begin{tabular}{lcccccccccc}
\hline \hline Bi & 1 & 2 & 3 & 4 & 5 & 6 & 7 & 8 & 9 & 10 \\
film & BL & BL & BL & BL & BL & BL & BL & BL & BL & BL \\
\hline$Z_{2}$ & 1 & 1 & 1 & 1 & 1 & 1 & 1 & 1 & 0 & 0 \\
\hline \hline
\end{tabular}

thin floating Bi bilayers, the bands of a Bi(111) surface layer have no band gap and no band inversion near the Fermi energy. As also shown in previous work [10], the surface layers of films become trivially metallic at a thickness larger than $4 \mathrm{BL}$, well below the thickness for the topological phase transition of the whole film. Therefore, there is no obvious reason a priori to expect any topological edge state on the edge of these surface layers. The origin of the difference between the floating $\mathrm{Bi}$ bilayer and the $\mathrm{Bi}(111)$ surface layer will be discussed further below.

We then calculated the edge band structure explicitly by simulating the step-edge structure of the surface layer on $\mathrm{Bi}(111)$ films of varying thickness. As shown in Fig. 1, we generate nanoribbons of 9-15 unit cells wide on top of $\mathrm{Bi}(111)$ films up to $9 \mathrm{BL}$ thickness. The thickness of $9 \mathrm{BL}$ is confirmed to be sufficient to simulate the topologically trivial bulk property as discussed above. Since all the atoms are freely relaxed, the edge atoms are substantially reconstructed to reduce the energy cost of the dangling bond creation. Two different edge configurations and reconstructions are formed due to the BL structure; edge atoms are buckled down or up for so-called A- or B-type edges, respectively, as shown in Fig. 1. These two edge structures yield slightly different edge state dispersions as shown in Fig. 3. These two edges have in common that the edge bands have Dirac-like crossings at the edge of the Brillouin zone $(X)$ within the enlarged band gap. These crossed bands have a helical spin texture (as confirmed in our spin-polarized calculations; data not shown here). This spin helicity and the Dirac-like crossings are indeed very similar to the QSH edge state of the 2D TI phase of a single BL film [Fig. 3(a)].

However, an important change can be noticed even for the 2 BL case, the nanoribbon on a single BL substrate [Fig. 3(b)]. Even at this thickness, the band gap of the film is reduced substantially, especially at the center of the Brillouin zone $(\Gamma)$, which closes down completely above the 8 BL substrate. An immediate consequence of the band-gap closure is that the topological nature of the step-edge state appears ambiguous even on the $1 \mathrm{BL}$ substrate; as the band gap closes, the upper and lower branches of the spin-split edge states get very close to each other at $\Gamma$ while their Dirac-like crossing at $X$ is preserved. If these two branches merge at $\Gamma$, then their dispersions become topologically trivial and the Dirac-like crossings at $X$ become trivial Rashba band crossings. This result is consistent with the previous calculation of the 2 BL edge with a much smaller nanoribbon width and with unrelaxed edge structures [20]. In fact, this change is what is expected from the topological phase transition of the film at a thickness between 8 and 9 BLs. For a thicker substrate than 4 BLs, the band-gap narrowing is substantial in the whole Brillouin zone and the edge states hybridize with the substrate 

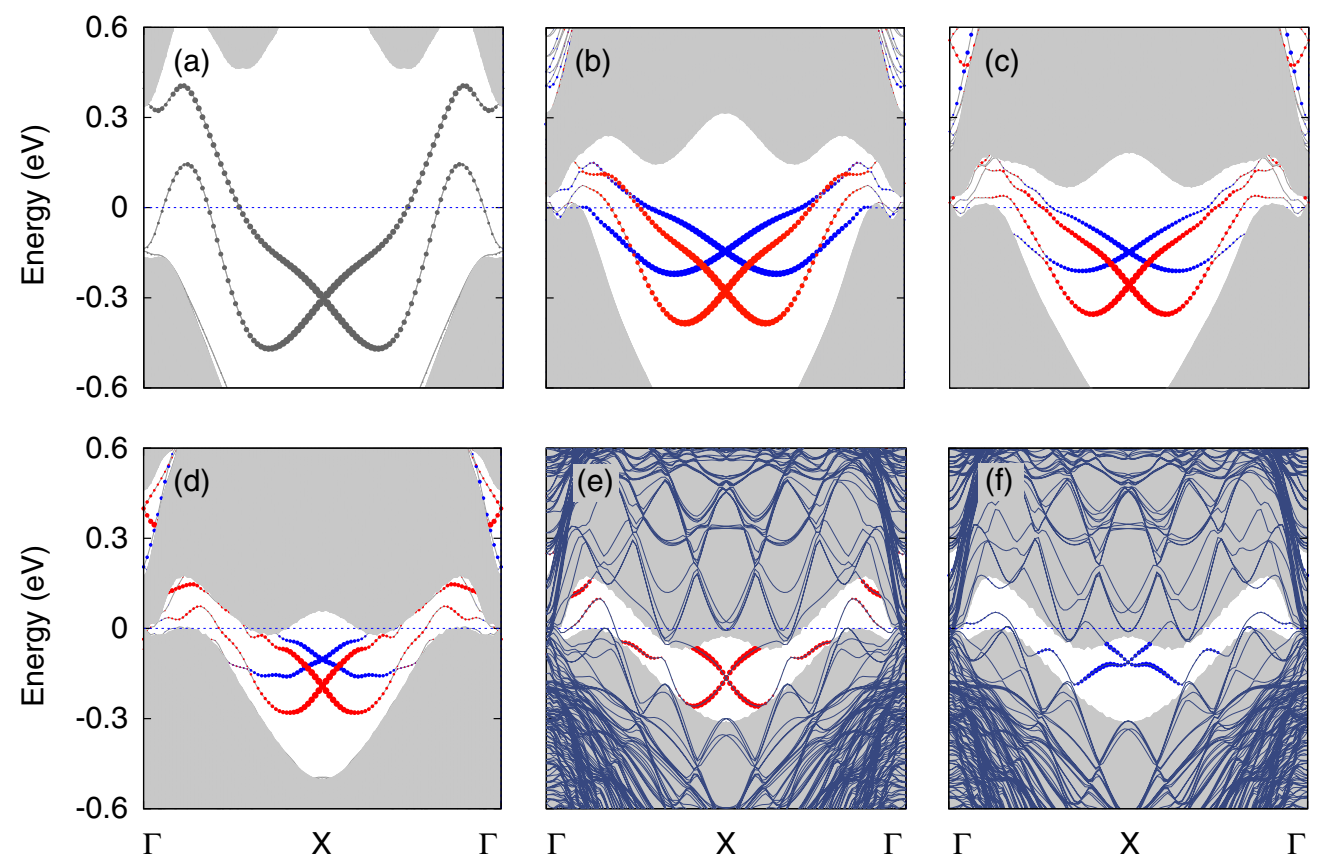

FIG. 3. Calculated band structure (a) of a pristine floating 1 BL Bi nanoribbon and of 1 BL Bi nanoribbons on top of (b) 1, (c) 2, (d) 4, and (e),(f) 9 BL films. Edge states are distinguished by red and blue dots for the A- and B-type edges according to Fig. 1. Shaded regions are the projection of the bulk (whole film) bands of 1, 3, 5, and 10 BL films, respectively.

bands for a large part of the Brillouin zone. Thus, the step-edge state is not truly localized along the edge atoms except for a limited part of the Brillouin zone. Note that these changes do not depend on the type of edge structures.

As mentioned above, the crucial part for the topological nature of the edge-state dispersion is the band dispersion near $\Gamma$. We thus scrutinize the change of the edge-state dispersions as shown in Fig. 4 for one type, type A, of the edge structure. As clearly shown here, the step-edge states on a sufficiently thick film have their spin-split bands merged at the $\Gamma$ point, becoming trivial Rashba spin-split bands. The same conclusion is reached for the other type of edges. We confirm that this change occurs concomitantly with the quantum phase transition from the 2D topological insulator to a 3D trivial semimetal. This can be shown by the disappearance of the band inversion between the bands of different $p$ orbitals; as depicted in Figs. 4(a) and 4(d), the valence and conduction bands at $\Gamma$ have a clear band inversion for the single floating BL film but return back to normal on the 9 BL substrate.

In contrast to the present work, the previous STS measurements appeared to show a topologically nontrivial step-edge state on top of a bulk $\mathrm{Bi}(111)$ crystal [12]. What was likely measured is a part of the dispersion of the edge-localized state, which is indicated by an arrow in Fig. 4(d). This part of the band had spin-polarized nature, due to the Rashba spin splitting. The topological nature of the edge states is, however, determined not by the spin splitting but by their dispersions especially at $\Gamma$ in the present case. While one can agree that these Rashba-type edge states evolve into QSH edge states at a very small thickness as already observed on single BL films on $\mathrm{Bi}_{2} \mathrm{Te}_{2} \mathrm{Se}$ and graphene [5-7], the bands in a thicker film and the bulk are obviously not topological edge states but 1D bands with Rashba spin splitting. The 1D chiral electron state itself with a large Rashba splitting was previously introduced in a surface nanowire array [21]. In other words, the strong coupling of the electronic states of a single BL surface layer
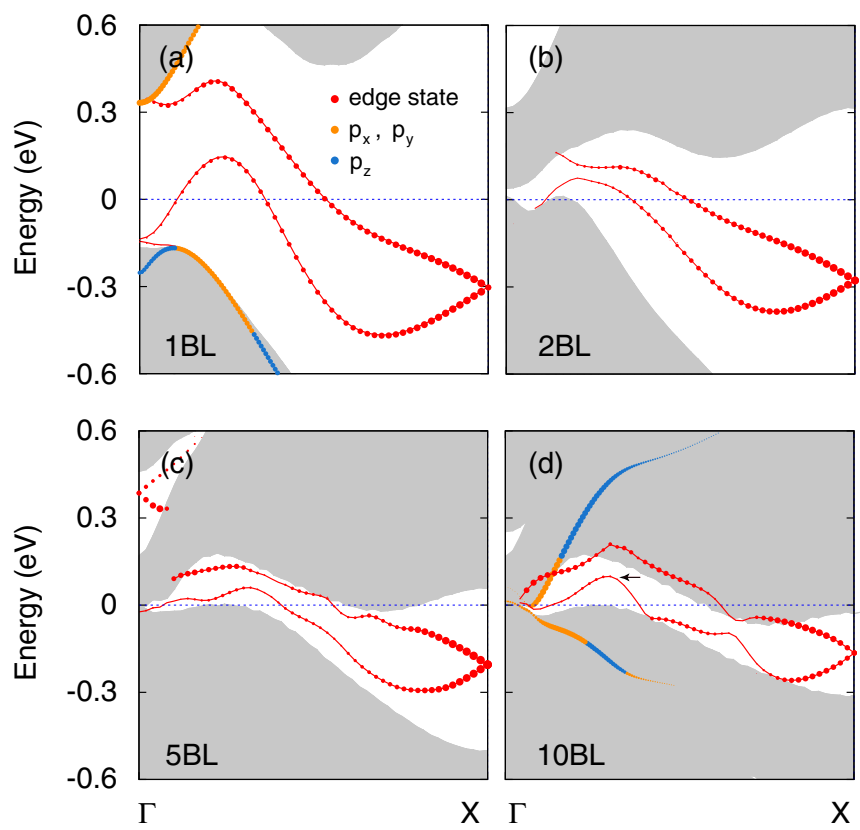

FIG. 4. Band structures of $\mathrm{Bi}$ nanoribbons on various $\mathrm{Bi}(111)$ thin films. (a) Edge states of a zigzag-edged floating Bi nanoribbon. (b)-(d) Edge states of the type-A edges (the same type of edge as in Ref. [12]) on 1, 4, and 9 BL films. Shaded regions are the projection of the corresponding bulk (whole film) bands. For the floating or surface layer of 1 and $10 \mathrm{BL}$ films, the orbital characters of the valence and conduction band edges are indicated. 

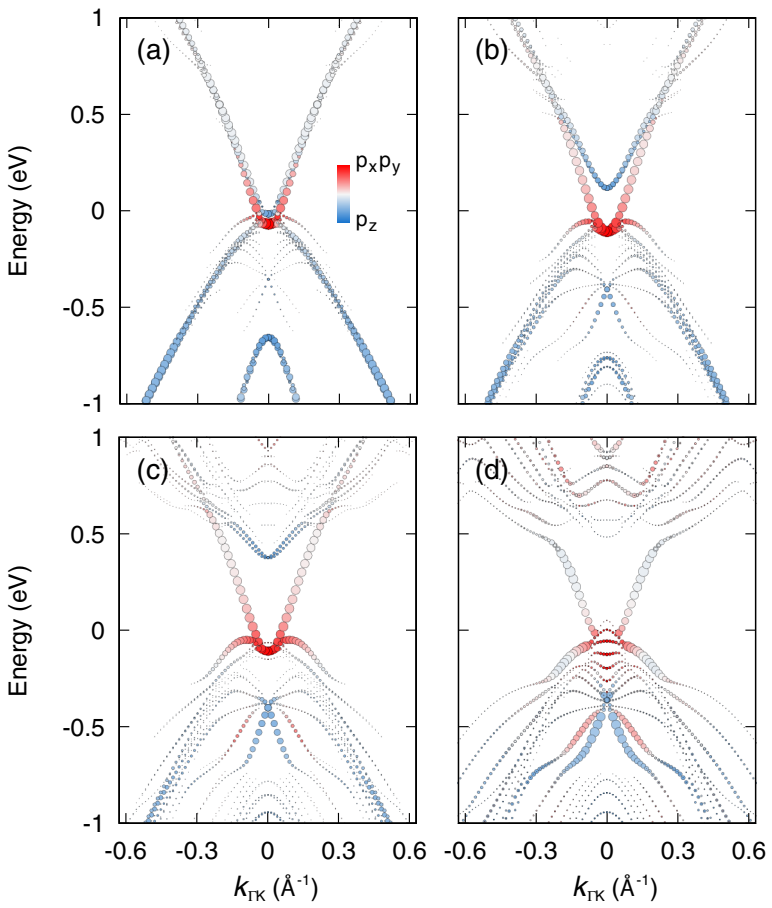

FIG. 5. (a)-(d) Calculated band structures of a single Bi bilayer on top of 9 BL films at a distance of $3,2,1$, and $0 \AA$ from the equilibrium distance, respectively. The states originating from the $\mathrm{Bi}$ bilayer are marked by red ( $p_{x}$ and $p_{y}$ orbitals) and blue ( $p_{z}$ orbital). The in-plane lattice constant is fixed to be that of the equilibrium surface layer case.

with those of its substrate destroys their topological nature. This substrate effect is detailed further in Fig. 5. We calculate the band dispersions of a single Bi BL at varying distances from its equilibrium position as the surface layer of $9 \mathrm{BL}$ film. Note that, even at a sufficiently large distance from the bulk with a minimal interaction, the single BL film loses the insulating property of a free-standing film. This is due to the substantial difference in lattice constant between the free-standing film and the bulk (about 5\% expansion in the bulk). Even with the closed band gap, the noninteracting film maintains its topological properties with the band inversion near the Fermi level as in the free-standing film. When the interaction with the bulk is turned on at a shorter distance, the band inversion disappears, with bands of a single character (red colored bands) across the Fermi level and the valence bands of the noninteracting film (blue bands) hybridized strongly with those of the bulk. These results show clearly how the single BL film and the $\mathrm{Bi}(111)$ substrate interact. The existence of a spin-helical edge state is necessary for a TI but not a sufficient condition to dictate the TI property and there is a substantial change of the electronic state of the Bi bilayer on the bulk substrate, not described in [12]

\section{CONCLUSION}

We investigated explicitly the topological nature of electronic states of step edges of $\mathrm{Bi}(111)$ films by first-principles calculations. We showed that the dispersion of step-edge states reflects the topological nature of the underlying films, rather than indicating the existence of topological edge states along step edges on surfaces of a bulk $\mathrm{Bi}(111)$ crystal or a sufficiently thick $\operatorname{Bi}(111)$ film. The trivial step-edge states have spin splitting of one-dimensional Rashba bands. This 1D band with a huge Rashba splitting might be interesting in the search for Majorana fermions but the existence of a strong intermixing with the substrate electronic state has to be considered. The topological character of an ultrathin 2D topological insulator put on a substrate has to be carefully examined, considering the strain and electronic hybridization with the substrate.

\section{ACKNOWLEDGMENT}

This work was supported by Institute for Basic Science (Grant No. IBS-R015-D1).
[1] B. A. Bernevig, T. L. Hughes, and S.-C. Zhang, Science 314, 1757 (2006).

[2] M. König, S. Wiedmann, C. Brne, A. Roth, H. Buhmann, L. W. Molenkamp, X.-L. Qi, and S.-C. Zhang, Science 318, 766 (2007).

[3] C. Liu, T. L. Hughes, X.-L. Qi, K. Wang, and S.-C. Zhang, Phys. Rev. Lett. 100, 236601 (2008).

[4] I. Knez, R.-R. Du, and G. Sullivan, Phys. Rev. Lett. 107, 136603 (2011)

[5] S. H. Kim, K.-H. Jin, J. Park, J. S. Kim, S.-H. Jhi, T.-H. Kim, and H. W. Yeom, Phys. Rev. B 89, 155436 (2014) and references therein.

[6] C. Sabater, D. Gosálbez-Martinez, J. Fernández-Rossier, J. G. Rodrigo, C. Untiedt, and J. J. Palacios, Phys. Rev. Lett. 110, 176802 (2013).

[7] Y. Lu, W. Xu, M. Zeng, G. Yao, L. Shen, M. Yang, Z. Luo, F. Pan, K. Wu, T. Das, P. He, J. Jiang, J. Martin, Y. P. Feng, H. Lin, and X.-s. Wang, Nano Lett. 15, 80 (2015).

[8] S. Murakami, Phys. Rev. Lett. 97, 236805 (2006).
[9] M. Wada, S. Murakami, F. Freimuth, and G. Bihlmayer, Phys. Rev. B 83, 121310 (2011).

[10] Z. Liu, C.-X. Liu, Y.-S. Wu, W. H. Duan, F. Liu, and J. Wu, Phys. Rev. Lett. 107, 136805 (2011).

[11] A. Takayama, T. Sato, S. Souma, T. Oguchi, and T. Takahashi, Phys. Rev. Lett. 114, 066402 (2015).

[12] I. K. Drozdov, A. Alexandradinata, S. Jeon, S. Nadj-Perge, H. Ji, R. J. Cava, B. A. Bernevig, and A. Yazdani, Nat. Phys. 10, 664 (2014).

[13] N. Kawakami, C.-L. Lin, M. Kawai, R. Arafune, and N. Takagi, Appl. Phys. Lett. 107, 031602 (2015).

[14] G. Kresse and J. Furthmüller, Phys. Rev. B 54, 11169 (1996).

[15] J. P. Perdew, K. Burke, and M. Ernzerhof, Phys. Rev. Lett. 77 , 3865 (1996).

[16] D. Hobbs, G. Kresse, and J. Hafner, Phys. Rev. B 62, 11556 (2000).

[17] A. A. Soluyanov and D. Vanderbilt, Phys. Rev. B 83, 235401 (2011). 
[18] Y. M. Koroteev, G. Bihlmayer, J. E. Gayone, E. V. Chulkov, S. Blügel, P. M. Echenique, and Ph. Hofmann, Phys. Rev. Lett. 93, 046403 (2004).

[19] Y. M. Koroteev, G. Bihlmayer, E. V. Chulkov, and S. Blügel, Phys. Rev. B 77, 045428 (2008).
[20] H. Kotaka, F. Ishii, M. Saito, T. Nagao, and S. Yaginuma, Jpn. J. Appl. Phys. 51, 025201 (2012).

[21] J. Park, S. W. Jung, M.-C. Jung, H. Yamane, N. Kosugi, and H. W. Yeom, Phys. Rev. Lett. 110, 036801 (2013). 